\title{
Urinary Infection -One of The Most Common Bacterial Infections in Clinical Practice
}

\author{
Angela Revelas ${ }^{1}$, Kyriakos Stefanidis ${ }^{2}$ \\ ${ }^{I}$ Pathological Department, St Nicolaos General Hospital-Crete, Greece \\ ${ }^{2}$ St. Paul General Hospital-Thessaloniki-Crete, Greece
}

Corresponding Author Angela Revelas Mobile:

6943503095

E mail: donnoiko@gmail.com

Urinary tract infection is one of the most common bacterial infections encountered in clinical practice. Most infections are uncomplicated. Complicated infections are caused by an abnormal urinary tract or a foreign body, the most common of which is catheter associated urinary tract infection.

\section{INTRODUCTION}

Urinary tract infections may be referred to as cystitis or pyelonephritis, terms that refer to the lower and upper urinary tract, respectively. The terms bacteriuria and candiduria describe bacteria or yeast in the urine. Very ill patients may be referred to as having urosepsis. Urinary tract infections occur more commonly in women than men, with half of women having an infection at some point in their life. Recurrences are common. Risk factors include sexual intercourse as well as a family history. Pyelonephritis usually follows a bladder infection but may also occur from a blood borne infection. Diagnosis in young healthy women can be based on symptoms alone. In those with vague symptoms, diagnosis can be difficult, because bacteria may be present without there being an infection. In complicated cases or if treatment has failed, a urine culture may be useful. In those with frequent infections, low dose antibiotics may be taken as a preventative measure. In uncomplicated cases, urinary tract infections are easily treated with a short course of antibiotics, although resistance to many of the antibiotics used to treat this condition is increasing. In complicated cases, longer course or intravenous antibiotics may be needed, and if symptoms have not improved in two or three days, further diagnostic testing is needed. In women urinary tract infections are the most common form of bacterial infection with $10 \%$ getting urinary tract infections yearly.

\section{PATHOPHYSIOLOGY}

The urinary tract is normally sterile. Uncomplicated urinary tract infections, involves the urinary bladder in a host without underlying renal, metabolic, or neurologic diseases. Cystitis represents bladder mucosal invasion, most often by enteric coliform bacteria (eg., Escherichia coli) that inhabit the periurethral vaginal introitus and ascend into the bladder via the urethra. Because sexual intercourse may promote this migration, cystitis is common in otherwise healthy young women. Generally, urine is a good culture medium. Factors unfavorable to bacterial growth include a low $\mathrm{pH}$ (5.5 or less), a high concentration of urea, and the presence of organic acids derived from a diet that includes fruits and protein. Organic acids enhance acidification of the urine. Most uropathogens gain access to ascending route. The shorter length of the female urethra allows uropathogens easier access to the 
bladder. The continuous unidirectional flow of urine helps to minimize urinary tract infections, and anything that interferes with this increases the host's susceptibility to urinary tract infections. Examples of interference include volume depletion, sexual intercourse, urinary tract obstruction, instrumentation, use of catheters not drained to gravity, and vesicoureteral reflux. Secretory defenses help promote bacterial clearance and prevent adherence. Secretory immunoglobulin A (IgA) reduces attachment and invasion of bacteria in the urinary tract. Women who are nonsecretors of the ABH blood antigens appear to be at higher risk for recurrent urinary tract infections. This may occur because of a lack of specific glycosyltransferases that modify epithelial surface glycolipids, allowing E. coli to bind to them better. In premenopausal women, lactobacilli are the predominant vaginal flora and serve to suppress vaginal colonization by the uropathogens.Most antibiotics, except sulfamethoxazole and the quinolones, can eradicate these protective bacteria. Urine itself has several antibacterial features that suppress urinary tract infections. Specifically, the $\mathrm{pH}$, urea concentration, osmolarity, and various organic acids prevent most bacteria from surviving in the urinary tract.

\section{MICROBIOLOGY OF UTI}

E.coli, is the cause of $80-85 \%$ of urinary tract infections, with Staphylococcus saprophyticus being the cause in $5-10 \%[1]$ Rarely they may be due to viral or fungal infections. [2] Other bacterial causes include. Klebsiella, Proteus, Pseudomonas, and Enterobacter. These are uncommon and typically related to abnormalities of the urinary system or urinary catheterization.[3] Urinary tract infections due to Staphylococcus aureus typically occurs secondary to blood infections.[4]

\section{CATHETERS}

Urinary catheterization increases the risk of urinary tract infections. The risk of bacteriuria, is between three to six percent per day and prophylactic antibiotics are not effective in decreasing symptomatic infections. [5] The risk of an associated infection can be decreased by catheterizing only when necessary, using aseptic technique for insertion, and maintaining unobstructed closed drainage of the catheter.[6,7]

\section{FAMILY HISTORY}

A predisposition for bladder infections may run in families. Other risk factors include diabetes, [8] being uncircumcised, and having a large prostate. [9] Complicating factors are rather vague and include predisposing anatomic, functional, or metabolic abnormalities. A complicated urinary tract infections is more difficult to treat and usually requires more aggressive evaluation, treatment and follow-up. In children urinary tract infections are associated with vesicoureteral reflux and constipation. [9]

\section{PROGNOSIS}

The prognosis for most women with cystitis and pyelonephritis is good. However, the prognosis for emphysematous pyelonephritis is not as good. Infected cysts in polycystic kidney disease respond to treatment slowly. Although simple lower urinary track infections may resolve spontaneously, effective treatment lessens the duration of symptoms and reduces the incidence of progression to upper urinary track infections. Younger patients have the lowest rates of morbidity and mortality. Factors associated with an unfavorable prognosis include.

- Old age

- General debility

- Renal calculi or obstruction

- Recent hospitalization

- Urinary tract instrumentation or antibiotic therapy

- Diabetes mellitus

- Chronic nephropathy

- Sickle cell anemia

- Underlying cancer

- Intercurrent chemotherapy

\section{IN CHILDREN}

In young children, the only symptom of a urinary tract infection may be a fever. Because of the lack of more obvious symptoms, when females under the age of two or uncircumcised males less than a year exhibit a fever, a culture of the urine is recommended. Infants may feed poorly, vomit, sleep more, or show signs of jaundice. In older children, new onset urinary incontinence may occur.[9] 


\section{IN THE ELDERLY}

Urinary tract symptoms are frequently lacking in the elderly. They may have vague presentations with incontinence, a change in mental status, or fatigue as the only symptoms. Diagnosis can be complicated by the fact that many elderly people have preexisting incontinence. Some elderly present to a health care provider with sepsis being the first symptoms [10].

\section{CATHETER-ASSOCIATED URINARY TRACT INFECTION}

Most patients with urinary tract abnormalities are referred to urologists. The closed urinary catheter system has a plastic bag fused to the distal end of a collection tube, allowing drainage so that the urine is always contained within a lumen closed to the environment. Even with meticulous attention to maintenance, the space between the external catheter and urethral mucosa provides an opportunity for direct entry of bacteria into the bladder. $10 \%$ to $30 \%$ of hospitalized patients with catheters develop bacteriuria. E.coli is the most common cause of catheter-related urinary tract infections, although it comprises only $25 \%$ of isolates. Other common causative organisms include Enterococci, Pseudomonas aeruginosa, K.pneumoniae, P.mirabilis, Enterobacter sp., Staphylococcus epidermidis, and Staphylococcus aureus. Candida may be isolated and appears to be increasing in prevalence, particularly when antibiotics are used .

Many modifications have made to the closed catheter systems, but most have not markedly improved the ability of the system to postpone bacteriuria. The use of systemic antibiotics has been more successful. Up to $80 \%$ of patients receive antibiotics during, but not usually because of, catheterization, and most studies have demonstrated a lower incidence of bacteriuria. When antibiotics are used. However, antibiotics are usually effective only for the first several days, after which resistant organisms begin to appear. Most authorities believe that antibiotics are not indicated for postponing bacteriuria in catheterized patients because of side effects, cost and emergence of antibiotic-resistant bacteria. However, antibiotics may be appropriate in patients at high risk for the complications of catheter-associated bacteriuria (eg., renal transplant recipients and granulocytopenic patients). Two catheter related principles are universally recommended:

-Keep the closed catheter system closed،

-Remove it as soon as possible.

Urine specimens should be obtained by needle and syringe, without opening the cathetercollection tube junction. Most catheter systems have parts on the distal catheter for needle aspiration of urine .If the catheter can be removed before bacteriuria develops, postponement of bacteriuria becomes prevention. Most episodes of short-term catheter-associated bacteriuria are asymptomatic. However, catheterassociated bacteriuria can be accompanied by fever, acute pyelonephritis, bacteremia, and death. In nursing homes, the incidence of catheter-associated bacteriuria is approximately the same as in hospitals. Because catheters in nursing home patients may be in place for months or years, almost all patients with indwelling catheters in nursing homes are bacteriuric. Indeed, polymicrobial bacteriuria is present in up to $95 \%$ of urine speciments from long-term catheterized patients. These colonizations are caused by common uropathogens, such as E. coli, P. aeruginosa, and $P$. mirabilis, as well as less familiar species, such as providencia stuartii and Morganella morganii .

Complications of long-term catheter-associated bacteriuria fall into two categories. The first category includes symptomatic urinary tract infections, such as those associated with shortterm catheterization(i.e., fever, acute pyelonephritis, bacteremia, and death).The second category includes complications associated primarily with long-term catheterization, such as catheter obstruction, urinary tract stones, local periurinary infections, chronic renal inflammation, renal failure, and after years of catheterization, bladder cancer. There are few data justifying the use of antibiotics in catheterized patients. However, antibiotic treatment is often necessary for symptomatic patients.In the catheterized patient who develops fever and/or signs of bacteremia, the clinician should rule out sources of infection outside the urinary tract, catheter obstruction, and periurethral infection, such as apidedimitis or prostatitis in men; blood and urine cultures should be obtained. Those patients should be empirically treated with parenteral antibiotics at doses high enough to achieve concentrations in serum that are adequate to treat bacteremia . 
Antibiotic selection should be based on knowledge of the organisms that are common in the medical unit and a Gram's stain of the patient's urine. Treatment should be modified on the basis of antibiotic susceptibilities. Seven to ten days of therapy is usually sufficient and may include oral administration. The occasional patient with bladder symptoms, such as lower abdominal pain without fever or other evidence of systemic infection, may, benefit from treatment with an oral antibiotic that is active against the causative organism in vitro. Because bacteria may be sequestered in a biofilm on the catheter surface, it may be reasonable to replace or remove the catheter. Candiduria may develop in catheterized patients, and the incidence is directly related to the duration of catheterization, hospitalization, and antibiotic use. The ideal approach to avoiding catheter-associated bacteriuria is to avoid catheterization .

Nursing care, patient training, and special garments and bed clothes can be used instead of catheterization in many situations. Other devices or techniques that assist in urinary drainage, including, condom catheters, intermittent catheterization, and suprapubic catheterization, should also be considered $[6,8,10]$.

\section{CONCLUSION}

Anyone with a long-term indwelling urinary catheter is at risk of developing a urinary tract infection (UTI). Using a catheter can introduce bacteria into the bladder and cause a UTI. The longer the catheter stays in the bladder, the greater this risk so that, after 30 days, bacteria will inevitably be present in the urine. This bacteriuria may or may not cause symptoms, but bacteria multiply quickly in the urine and the constant friction of an indwelling catheter on the lining of the bladder ulcerates its surface, enabling the bacteria to invade the bladder wall and blood stream. From the bladder, infection can spread up the tubes or ureters to the kidneys. The catheter is held in the bladder by a balloon, which is filled with sterile water, and this prevents the bladder from emptying completely so that you always have some residual urine in the bladder, and that will be infected by bacteria. Secondly, the catheter has a protuberant tip and this can damage that surface lining in the bladder and the eye holes are in the tip of the catheter, not at the base of the bladder. So we have here a problem which does mean that the catheter is associated with a very high incidence of healthcare infections. In fact it accounts for more healthcare infections worldwide than any other medical device.

Proper adherence to the outpatient medical regimen should be stressed. Behavior modification such as good oral fluid intake to enhance diuresis, frequent voiding, and drinking fruit juices to acidify the urine are helpful in reducing recurrent infection.

\section{REFERENCES}

1. Nicolle LE. Uncomplicated urinary tract infection in adults including uncomplicated pyelonephritis. Urol Clin North Am. 2008;35(1):1-12.

2. Amdekar S, Singh V, Singh DD. Probiotic therapy: immunomodulating approach toward urinary tract infection. Current microbiology 2011;63(5):484-90.

3. Salvatore S, Cattoni E, Siesto G, Serati M, Sorice P, Torella M. Urinary tract infections in women. Eur J Obstet Gynecol Reprod Biol. 2011; 156 (2): $131-6$

4. Lane DR, Takhar SS.Diagnosis and management of urinary tract infection and pyelonephritis. Emerg Med Clin North Am. 2011; 29 (3), 539-52.

5. Bhat RG; Katy TA. Place FC "Pediatric urinary tract infections. Emerg Med Clin North Am. 2011;29 (3): 637-53

6. Nicolle LE. The chronic indwelling catheter and urinary infection in long-term- care facility residents. Infect Control Hosp Epidemiol 2001;22[5]:316-21

7. Gould CV, Umscheid CA, Agarwal RK., Kuntz G. Guidline. for prevention of catheterassociated urinary tract infections 2009. Infect Control Hosp Epidemiol. 2010;31(4):319-26.

8. Nicolloe LE. A practical approach to the management of complicated urinary tract infection.Urol Clin North Am 2008; 35:1-12. 3.

9 Bhat RG., Katy TA., Place FC. Pediatric urinary tract infections Emerg Med Clin North Am. Aug 2011;29(3):637-58.

10. Lane DR, Takhar SS. Diagnosis and management of urinary tract infection and pyelonephritis. Emerg Med Clin North Am. 2011;29(3):539-52.

Peer reviewer: Emad F Hamed: Lecturer of Internal Medicine, Zagazig University, Egypt.Editor: Mohamed Emara: Lecururer of Tropical Medicine, Zagazig University, Egypt. 